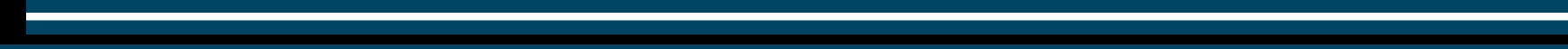




\section{La formación del Arquitecto paisajista Colombiano $^{1}$}

\section{The colombian \\ landscape architect education}

\section{Resumen:}

\section{Palabras clave:}

Arquitecto Diseñador del paisaje, paisaje, educación, saberes, capacidades.

\section{Key words:}

Landscape Architect, landscape, education, knowledge, abilities.

Recibido: 05 dic. de 2011 Aceptado: 24 ago. de 2012

*Arquitecta, Magíster en Diseño del Paisaje de la Universidad Pontificia Bolivariana de Medellín. Profesora asistente de la misma Universidad. Integrante del grupo de investisación LAUR (Laboratorio de Arquitectura y Urbanismo)

${ }^{1}$ Texto soporte de la ponencia que con el mismo nombre fue presentada en octubre de 2011 en el marco del seminario "Repensando el paisaje de la ciudad del siglo XXl", realizado en la Universidad de Boyacá.

Juliana.cadavid@upb.edu.co
Este documento, correspondiente a una reflexión no derivada de investigación, integra una serie de consideraciones respecto al papel del arquitecto-diseñador del paisaje colombiano, su formación y su acción. El texto se estructura a partir de preguntas acerca de la pertinencia del ejercicio de esta profesión y de las capacidades generales que debe tener un arquitecto paisajista en nuestro medio para afrontar las responsabilidades que implica su ejercicio en el contexto colombiano. Seguidamente se expone el programa de Maestría en Diseño del Paisaje de la Universidad Pontificia Bolivariana de Medellín, como una apuesta académica y formativa ante la creciente necesidad de profesionales del paisaje en nuestro medio. Se hace referencia a su estructura, objeto de formación, currículo y metodologías que el egresado ha de aplicar en su futuro desempeño, las cuales se orientan al entendimiento, diagnóstico e intervención en el paisaje. Todo ello se enmarca en un contexto de integración de múltiples disciplinas asociadas al paisaje.

\section{Abstract}

This paper explains considerations regarding the role of the Colombian landscape architect, his education and action. The text presents a reflection of the skills that a landscape architect should have in our environment, and the responsibilities he needs to face in his profession, specifically in the Colombian scene. Also the Masters in Landscape Design at the Universidad Pontificia Bolivariana at Medellín in Colombia is displayed, as an academic commitment to face the growing need for landscape professionals in our country. The master's aspects discussed are its structure, object of training, curriculum and methodologies of intervention the graduate should apply in future performance; which are oriented to understanding, diagnosing and intervening landscape. All of this centered on the role inherited to the landscape architect of being an integrative mediator between different disciplines. 
Es importante empezar esta reflexión preguntándonos: ¿por qué es pertinente la disciplina de la Arquitectura del Paisaje en Colombia? Habitamos un pás diverso, tanto desde el punto de vista natural como desde el humano. Nuestra situación geográfica nos otorga el privilegio de poseer una rica variedad de flora y fauna. La geografía, la vida desarrollada en ella y la interacción entre ecosistemas, son realidades que nos identifican y definen nuestra convivencia cotidiana con este entorno magnífico que nos marca los afectos. Así, se deriva una cultura estrechamente arraigada con el territorio habitado.

Esa relación geográfica, biológica y cultural define la idea de paisaje. Hemos construido una infinidad de imásenes de nuestro territorio a las que denominamos con este concepto. Al mismo tiempo, cada marca física y distinción espacial es un trozo de memoria colectiva producto de la experiencia.

A su vez, la diversidad nos caracteriza. Nuestra cultura es la sumatoria de muchas. ¿Cuántos paisajes hemos construido sobre la base de este generoso entorno? Esta interacción genera un sentido estético propio, soportado en los afectos, en lo que hemos aprendido a ver y sentir como bello. Por tal razón, el sentir de la cultura en relación con el paisaje construido y habitado ha de primar frente a cualquier intervención sobre el territorio. Al respecto, es preciso superar el funcionalismo prasmático y evitar el esteticismo romántico y sin sentido. Y en tal dirección, el diseño del paisaje juega un papel fundamental. 
Podría afirmarse que un territorio como el nuestro debe contar con profesionales cuya mirada esté formada en función de valorar y potenciar la riqueza paisajística, y sobre todo, que posean las capacidades apropiadas para velar por una correspondencia entre las intervenciones en el territorio y la identidad de los mismos.

Así, cuando se habla del paisaje se piensa también en territorio, un concepto difícil de enmarcar en términos de límites políticos, dada su relación con fenómenos que trascienden las normas y abarcan múltiples escalas. Por lo tanto, podemos afirmar que nuestro territorio, con todas sus cualidades paisajísticas, requiere arquitectos y diseñadores, como también profesionales de distintas disciplinas, formados para actuar en un entorno específico.

\section{¿QUÉ CAPACIDADES DEBE TENER UN ARQUITECTO DEL PAISAJE EN COLOMBIA?}

Un arquitecto o diseñador del paisaje debe estar formado tanto en el conocimiento como en la acción. Esto implica que su educación, en lugar de estar perfilada hacia la especialización, ha de ser lo suficientemente amplia para desenvolverse en los múltiples campos asociados con la intervención paisajística. Entendemos que, al ser una disciplina relativamente nueva en Colombia, el primer llamado estará enfocado en la interacción de este profesional con diversos ámbitos.

Así, mientras el ejercicio del diseño está directamente vinculado con lo físico-espacial, el papel del arquitecto o diseñador paisajista se remitirá, en primera medida, a posicionar su actividad, gestionar su importancia y convertirse en un profesional indispensable frente a cualquier intervención del territorio, independientemente de la escala de dicha acción.

\section{La interacción de disciplinas en la Arquitectura del Paisaje}

La Arquitectura del Paisaje está orientada a estructurarse con base en los principios de áreas más especializadas. Esto no significa, de manera alguna, que el profesional de este campo se encuentre en una posición de competir específicos, los cuales son dispuestos en función de un objeto general como es la transformación del territorio, la intervención en el paisaje.

En una posición intermedia e integradora entre las ciencias naturales y las ciencias sociales, la Arquitectura del Paisaje, como mirada analítica y proyecto de intervención del territorio, constituye una base conceptualmente articulada, sinérgica y superadora de los enfoques tradicionales que han caracterizado el accionar sobre la ciudad, el medioambiente y sus problemáticas. (Moreno, 2008,p. 1). 
La Arquitectura del Paisaje puede entenderse como una mirada unificadora que comprende distintos horizontes, dirigidos a la interpretación del territorio. Simultáneamente, está en capacidad de articularlos en la función final de intervención, cuando esto sea oportuno. Sus categorías disciplinares se integran con diversas perspectivas filosóficas y técnicas: la estética y la teoría están en interacción con la Geología y la Hidrología, al tiempo que mantienen un diálogo con otras áreas, como la Ecología del Paisaje o la Antropología.

Se busca dejar claro que, de manera contraria a la vocación especializada de las disciplinas propias de la postmodernidad, la Arquitectura del Paisaje actúa como integradora de las múltiples variables que deben tenerse en cuenta al momento de intervenir no solo el espacio físico, sino también las acciones bióticas y culturales que se manifiestan en un lugar y tiempo determinados.

\section{La gestión de la disciplina del paisaje}

Como un objetivo propio de una disciplina encaminada a abrirse campo en un contexto en que aún no ha sido integrada apropiadamente, el arquitecto paisajista está llamado a la gestión, tanto del reconocimiento de su actividad como de la transformación de las leyes y políticas involucradas con el paisaje.

\section{En Colombia, la legislación considera tangencialmente al paisaje, si bien confiere} cierto énfasis a los paisajes culturales asociados con valores patrimoniales. Nuestra normativa se ha centrado en definir usos del suelo, áreas rurales de protección, disposiciones acerca de la planificación de zonas urbanas o urbanizables, más no especifica lineamientos precisos frente al paisaje. En general, éste no es entendido como un asunto integral, ya que sus elementos son considerados por separado. En consecuencia, tal vez el primer lazo a construir será el relacionado con el vínculo hombre - entorno - hábitat.

El hecho de que los marcos de actuación sobre el territorio, ya sea a través de normas nacionales (Leyes 99 de 1993, 142 de 1994) o disposiciones municipales (Planes de Ordenamiento Territorial) no consideren el tema del paisaje como directriz, nos obliga a continuar con visiones segmentadas frente al territorio y la cultura, y por ende, a ofrecer soluciones parciales.

Esta condición se evidencia en la mirada atomizada del espacio, en los conflictos entre elementos que hoy afectan al paisaje y lo presentan fragmentado. Ejemplos de tal realidad se registran en los bordes urbano-rurales de nuestras ciudades, en las enormes áreas de cultivos extensivos, en cada hidroeléctrica construida. Estos son lugares de conflicto y disputa donde se conjugan las principales problemáticas del paisaje. 
Si bien estas y muchas otras situaciones no son exclusivas de Colombia, se manifiestan con mayor vehemencia en países subdesarrollados y en vías de desarrollo -como el nuestro- que paradójicamente, poseen mayor riqueza en cuanto a condiciones naturales.

Frente a esta miopía de la legislación podemos asirnos a otras cartas de navegación, específicamente a las declaratorias de la Unesco, el Convenio Europeo del Paisaje y por supuesto, la Carta Colombiana del Paisaje. Estos documentos indican como en varios lugares del mundo se hacen constantes esfuerzos por defender el paisaje y considerar su papel integrador, el cual no solo responde a políticas de crecimiento económico, sino que asume la economía como un elemento más.

Más que adoptar una visión estética, se trata de considerar los factores involucrados en el espacio y el tiempo y buscar la significación y el respeto de los componentes humanos y naturales del paisaje, como también de su constante interacción con las condiciones impuestas por un mundo globalizado.

\section{La educación}

Las transformaciones requieren tiempo. Una tarea fundamental que debe cumplir el arquitecto del paisaje es educar, pues transmitir sus conocimientos a las nuevas generaciones es garantía de cambios futuros. La educación en este contexto implica asumir la disciplina paisajística como una mirada orientada a abarcar el espacio de manera integral. Por consiguiente, considera que las posibles intervenciones en un territorio han de ser ante todo formadoras, tanto del entendimiento como de las acciones. Sensibilizar, más que adoctrinar.

Claro está, es preciso evitar el error de creer que cada miembro de la sociedad debe tener un concepto y una responsabilidad intelectual frente al paisaje: esto iría precisamente en contra de la diversidad. La tarea del arquitecto paisajista, por medio de su gestión, su interpretación de los entornos o de las mismas transformaciones físicas, es transmitir al usuario-habitante un mensaje a partir del espacio. Si éste se encuentra debidamente articulado con lo natural y con lo cultural, será apropiado, estético y al mismo tiempo educador.
1. Una literatura (oral o escrita) que cante la belleza de los lugares; 2. Jardines de recreo; 3 . Una arquitectura dispuesta para disfrutar de una bella vista; 4 .
Pinturas que representen el medio ambiente $; 5$. Una o varias palabras para decir "paisaje"; 6. Una reflexión explícita sobre el paisaje. (Berque, 2008, citado por Saldarriaga, 2011, p. 123) 
Para ser consecuentes con los criterios de Berque, en cuanto a la reflexión explícita sobre el paisaje tenemos vacíos. De hecho, podría afirmarse que en nuestro medio, la ausencia de una reflexión propia ha impedido involucrar la disciplina a los campos y estamentos transformadores del territorio.

En el contexto latinoamericano, los académicos de la Geografía Humana han desarrollado un pensamiento en torno al paisaje. Entre ellos se destaca Milton Santos, quien ha construido, a partir de la metodología investigativa, una mirada teórica estrechamente relacionada con la geografía y la sociedad:
No basta con proclamar que el espacio geográfico existe como un dato inseparable del resto de la vida social. Los lugares y las regiones se vuelven fundamentales para explicar la producción, el comercio, la política, por lo que es imposible dejar de reconocer su papel en la elaboración del destino de los países y del mundo. El espacio geográfico se transforma en algo dotado de gran autonomía en el proceso histórico, y es realmente este hecho -esa madurez histórica- lo que lleva a una reafirmación de la geografía en el rol de los saberes (...) El territorio, tomado como un todo dinámico, es hoy el principal revelador de los grandes problemas nacionales, ya que permite una visión fragmentada y unificada de los diversos procesos sociales, económicos y políticos. (Santos, citado por Bosque \& García, 2003, p. 15).

Esta tendencia hacia un enfoque integrador del territorio está ligada a la slobalización, sobre la que Santos es reiterativo en sus consideraciones. Precisamente, este fenómeno despierta la necesidad de entender las identidades, de actuar frente a lo genérico que se posiciona en nuestra realidad como una serie de modelos de transformación del espacio completamente ajenos a la cultura y la historia propias.

Por ello, es fundamental una reflexión filosófica profunda, destinada a sentar las bases de una ética que se anteponga a las acciones actuales o al menos las cuestione, y que permita prever lo hecho hasta hoy y cuánto debemos hacer en el futuro. La aplicación de modelos ajenos en la intervención del territorio ha llevado a errores, la mayoría de ellos evidenciados como acciones caprichosas que enferman los espacios. Esta es una consecuencia directa de la falta de atención respecto al verdadero significado de nuestro propio entorno. Así, la reflexión metodológica, sistémica y responsable podrá orientar el futuro y llevará a concebir el paisaje como el trasfondo filosófico bajo el cual se entiende la significación de nuestro territorio. 


\section{¿QUÉ DEBE SABER UN ARQUITECTO DEL PAISAJE COLOMBIANO?}

Tras la contextualización del saber y el ejercicio del arquitecto paisajista en función de sus capacidades y sus campos de acción, se explica a continuación la estructura de la Maestría en Diseño del Paisaje de la Universidad Pontificia Bolivariana, sede Medellín. Este programa surge como una apuesta por la formación integral de profesionales capacitados en esta área del conocimiento, pues se posee una férrea convicción acerca de la necesidad de su labor. Se trata de una propuesta sustentada en una postura ética, que desde la academia aborda la lectura, reflexión y transformación del territorio.

Antes de describir la orientación y el plan de estudios, es importante aclarar que este programa se ha denominado "Diseño" y no "Arquitectura" del paisaje, precisamente en vista de la necesidad de integrar disciplinas distintas a la Arquitectura. El sencillo cambio de términos ha permitido que el programa forme en la disciplina del paisaje a profesionales de diversos campos, tales como biólogos, abogados, ingenieros forestales, y por supuesto, arquitectos, con el agregado de que muchos de ellos se desempeñan total o parcialmente en la educación y la investigación.

${ }^{2}$ Primer programa de Maestría, el cual reconoce como antecedente el importante papel que han desempeñado otras propuestas en el país, aprobadas en el nivel de especializaciones. Además, tiene presente los aportes en la disciplina de diferentes grupos y líneas de investigación del paisaje, pertenecientes a reconocidas instituciones educativas de todo Colombia.

\section{Programa de Maestría en Diseño del Paisaje de la Universidad Pontificia Bolivariana} Presentado como el primero de esta disciplina en Colombia ${ }^{2}$, se propone impulsar en el país y particularmente en la región, un campo del conocimiento que ya es amplio y sólido en otras partes del mundo. Se caracteriza por una visión de conjunto del lugar geográfico, histórico, cultural y político en donde actúan las generaciones del presente para desarrollar hábitats enriquecedores y sostenibles. Tal orientación conlleva a enfatizar en la interdisciplinariedad y la integridad (Universidad Pontificia Bolivariana [UPB], 2007). 
La conformación curricular abarca cuatro semestres, durante los cuales los estudiantes cumplen un total de 676 horas presenciales y comprometen al menos 1820 horas de trabajo autónomo. Con este tiempo se busca formar al educando por medio de una metodología que inicia con la fundamentación en primer semestre, pasa a la exploración en segundo, prosigue con la integración en tercero y llega a la profundización en cuarto. De tal modo, la investigación se convierte en el soporte para el diseño del paisaje.

Esta estructura, lineal en el tiempo, se involucra de forma transversal a través de los cuatro componentes fundamentales de la disciplina del paisaje: lo natural, lo humano, lo funcional y lo teórico. Éstos, a su vez, se conjugan en un quinto componente que es la integración.

El programa completo se orienta hacia un proceso progresivo de aprendizaje que permite al alumno comprender, explorar y practicar la disciplina.

Sus egresados son definidos como profesionales competentes para actuar en equipos multidisciplinarios, principalmente como líderes, coordinadores o asesores, con conocimientos y habilidades suficientes para lograr la integralidad que haga de los trabajos a su cargo, proyectos paisajísticos responsables, aportantes a la calidad del hábitat de la ciudad o región en la que se desempeñen". (UPB, 2007, p. 28).

En este sentido, la Maestría apunta a los requerimientos del medio, no solo en términos de preparar a los estudiantes para diversos campos, sino también para integrar en ellos las premisas expresadas en el perfil citado.

\section{Estructura de la Maestría}

Como se aprecia en la figura 1, la formación se define por ciclos. De esta forma, los componentes que fundamentan la estructura adoptan un carácter transversal en los cuatro semestres y se integran a lo largo del currículo.

\section{ENFOQUE DEL PROGRAMA $C O M P O N E N T E S$} Natural Humano Funcional Técnico Intgración
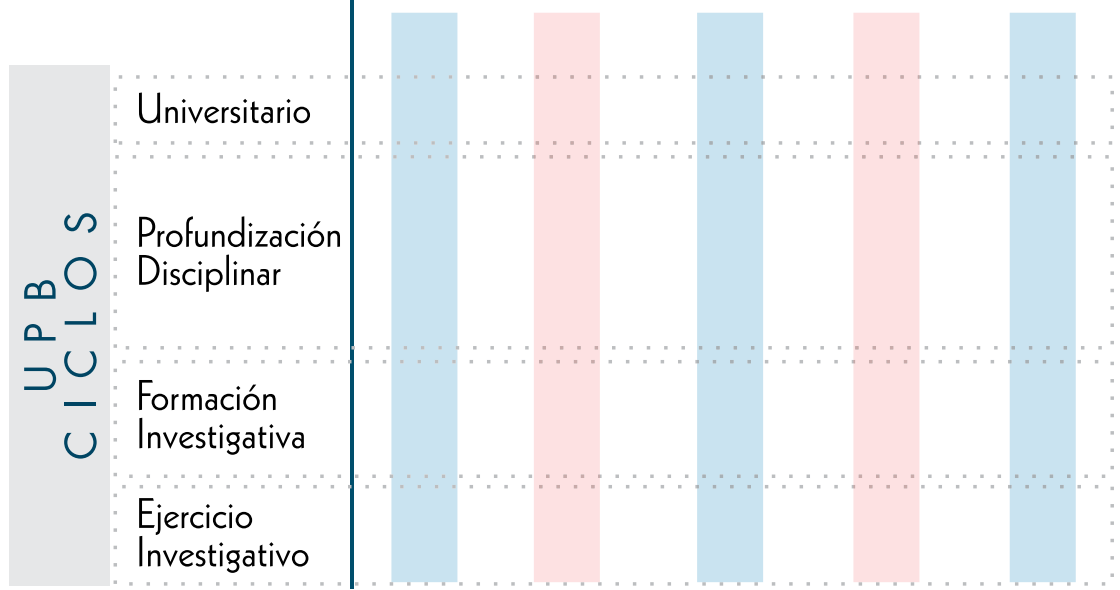

Fuente: UPB, 2007, P. 34 
Los diferentes componentes, con los respectivos cursos que integra cada uno de ellos, pueden presentarse como sigue:

\begin{tabular}{|c|c|}
\hline COMPONENTES & CURSOS \\
\hline Natural & $\begin{array}{l}\text { Ecología del Territorio. Hidrología, Vegetación, } \\
\text { Flora Local. }\end{array}$ \\
\hline Humano & $\begin{array}{l}\text { Historia y Teoría del Paisaje, Percepción y Recurso } \\
\text { Visual, Paisaje Antioqueño, Gestión Comunitaria, } \\
\text { Legislación ambiental y del Paisaje }\end{array}$ \\
\hline Funcional & $\begin{array}{l}\text { Naturaleza y ciudad, Sistemas Ürbanos, Parques } \\
\text { y Jardines }\end{array}$ \\
\hline Técnico & $\begin{array}{l}\text { Representación, Construcción del Paisaje, Sistemas } \\
\text { de Información, Manejo de la Vegetación, } \\
\text { Geoingeniería, Landcad }\end{array}$ \\
\hline
\end{tabular}

Asimismo, el Momento de Integración abarca los cursos: Ética Ambiental, Análisis del Paisaje, Metodología de Diseño, Investigación y Talleres de diseño.

Metodológicamente, los ciclos integran los componentes en función de la intervención en el paisaje. Lo aprendido en cada uno debe ser aplicable en el ejercicio del diseño en los talleres. Éstos adquieren complejidad en la medida que el estudiante avanza en los semestres y logra la capacidad de aplicar metodologías del diseño del paisaje en todas las etapas: lectura, diagnóstico, definición de criterios de intervención y desarrollo espacial de las propuestas.

Conjuntamente con el proceso académico formativo, el programa está vinculado con asociaciones del paisaje nacionales e internacionales. La Sociedad Colombiana de Arquitectos Paisajistas (SAP) ha contribuido a partir de la participación de sus miembros en la docencia y la organización compartida de eventos. Igualmente, se han coordinado encuentros de educación en asocio con la International Federation of Landscape Architects $^{3}$ (IFLA). 
En múltiples espacios en que el paisaje es tema central, la Maestría ha sido ampliamente representada desde el momento mismo de su formulación. Estas son acciones de relación y reflexión de gran importancia para la consolidación de la disciplina en nuestro medio.

De manera paralela con la formación de arquitectos paisajistas, el programa busca involucrar a los educandos con diferentes redes, pues así, además de su educación, obtienen un panorama macro del ejercicio disciplinar en contextos diversos. Este es otro conducto para sembrar en los nuevos profesionales la necesidad de trabajar en comunidad y desde la interdisciplinariedad.

\section{CONCLUSIONES}

Más que una presentación de un programa académico de posgrado, se ha buscado plantear una reflexión acerca del papel que la Arquitectura del Paisaje debe representar en un medio como el nuestro.

En este documento es reiterativo el llamado a los arquitectos - diseñadores del paisaje para que asuman, desde su propio compromiso ético y su quehacer profesional, el papel de ser a la vez gestores y transformadores de su territorio. Para tal fin, resulta esencial la visión integradora inculcada en su formación, como también el convencimiento de que su trabajo ha de ser educador. Así, sus obras podrían propiciar una transformación conducente a una cultura respetuosa y consciente de su entorno. 


\section{REFERENCIAS \\ BIBLIOGRÁFICAS}

Bosque, J. \& García, A. (2003). Milton de Almeida Santos (1926 - 2001). Anales de Geografía, 23, 9-19.

Moreno, O. (2008). La Arquitectura del Paisaje: retrospectiva y prospectiva de la disciplina a nivel global y latinoamericano. Enfoques, tendencias, derivaciones. Trabajo presentado en el Seminario Taller La enseñanza del Paisaje en grado y postgrado, Santiago, Chile.

Saldarriaga, A. (2011). Buscando el paisaje en el Valle de Aburrá. Bitácora, 16, $121-116$.

Universidad Pontificia Bolivariana (2007). Maestría en Diseño del Paisaje. Informe de condiciones de calidad con fines de obtener el registro calificado. Medellín, Colombia: el autor. 